\title{
RNAi for contactin 2 inhibits proliferation of U87-glioma stem cells by downregulating AICD, EGFR, and HESI
}

This article was published in the following Dove Press journal:

OncoTargets and Therapy

13 February 2017

Number of times this article has been viewed

\author{
Yang Guo',* \\ Peidong Zhang ${ }^{2,3, *}$ \\ Hongtian Zhang ${ }^{4}$ \\ Peng Zhang ${ }^{4}$ \\ Ruxiang $\mathrm{Xu}^{4}$ \\ 'Department of Neurology, \\ ${ }^{2}$ Department of Cardiovascular \\ Medicine, Zhujiang Hospital, ${ }^{3}$ Second \\ Clinical Medical College, Southern \\ Medical University, Guangzhou, \\ ${ }^{4}$ Department of Neurosurgery, \\ Affiliated Bayi Brain Hospital, The \\ Military General Hospital of Beijing \\ PLA, The Bayi Clinical Medical \\ Institute of Southern Medical \\ University, Beijing, People's Republic \\ of China
}

*These authors contributed equally to this work
Correspondence: Ruxiang Xu

Department of Neurosurgery, Affiliated Bayi Brain Hospital, The Military General Hospital of Beijing PLA, The Bayi Clinical Medical Institute of Southern Medical University, Beijing 100000, People's

Republic of China

$\mathrm{Tel}+8613391788118$

Fax +8602062783083

Email sjnd053@163.com

\begin{abstract}
Glioblastoma is the most common form of malignant brain tumors and has a poor prognosis. Glioma stem cells (GSCs) are thought to be responsible for the aberrant proliferation and invasion. Targeting the signaling pathways that promote proliferation in GSCs is one of the strategies for glioma treatment. In this study, we found increased expression of contactin 2 (CNTN2) and amyloid $\beta$ precursor protein (APP) in U87-derived GSCs (U87-GSCs). RNA interference (RNAi) for CNTN2 downregulated the expression of APP intracellular domain (AICD), which is the proteolytic product of APP. Treatment with CNTN2 RNAi inhibited the proliferation of U87-GSCs. CNTN2 RNAi decreased the expression of epidermal growth factor receptor and HES1, which are potential targets of AICD. In summary, inhibition of the CNTN2/APP signaling pathway may repress the proliferation in U87-GSCs via downregulating the expression of HES1 and epidermal growth factor receptor. CNTN2/APP/AICD signaling pathway plays an important role in U87 glial tumorigenesis. Further studies are warranted to elucidate the role of these signaling pathways in other sources of GSCs. Depending on their role in proliferation in other sources of GSCs, members of the CNTN2/APP/AICD signaling pathway may provide novel targets for the development of therapy for glioblastomas.
\end{abstract}

Keywords: contactin 2, CNTN2, transient axonal glycoprotein-1, TAG1, glioma stem cells

\section{Introduction}

Glioma is the most common primary tumor of the central nervous system. It accounts for $80 \%$ of malignant brain tumors in the US. ${ }^{1}$ Glioblastoma multiforme (GBM) represents the most malignant form and accounts for $46 \%$ of malignant brain tumors in the US. ${ }^{1}$ The standard treatment for glioma is surgical resection followed by radiotherapy, chemotherapy, or biotherapy. Despite improved therapies, the prognosis of patients remains poor. The ongoing research suggests that the genesis, development, and relapse of gliomas may originate from the aberrant proliferation of a subpopulation of cells, the glioma stem cells (GSCs). GSCs can proliferate indefinitely, self-renew, and initiate tumor, which make them different from differentiated tumor cells. They are becoming a main target for the treatment of gliomas. ${ }^{2-4}$

Though it is not clear whether the GSCs originate from neural stem cells (NSCs) or differentiated astrocytes, all glioma cells have the potential to develop stemness, depending on their microenvironment. ${ }^{5}$ The U87 cell is an established cell line from a male patient with grade IV glioblastoma with known genome sequence. ${ }^{6}$ Recently, a U87-derived GSC (U87-GSC) subline has been isolated and has become a broadly used model to study the pathological mechanisms and pharmacological therapy of 
gliomas $^{7-14}$ due to its stemness characteristics of self-renewal, high proliferative potential, and tumor initiation capacity. ${ }^{3}$

Targeting GSC signaling pathways or niche for proliferation is one of the strategies for glioma treatment. ${ }^{15}$ Understanding the molecular mechanisms of GSC development is essential to identify novel molecular targets for the treatment of gliomas. Previous studies with U87-GSCs or other GSCs suggest that GSCs share many characteristics with NSCs, including expression of Nestin and CD133 and activation of Notch, epidermal growth factor receptor (EGFR), JAK/STAT, PI3K/AKT, and mGlu3 receptor signaling pathways. ${ }^{7,8,10,14,16-19}$ Activation of Notch pathway is correlated with the chemoresistance or radioresistance of GSCs. ${ }^{17}$ A recent study in NSCs demonstrates that transient TAG1, or contactin 2 (CNTN2) as a functional ligand of amyloid $\beta$ precursor protein (APP) can trigger the $\gamma$-secretase-dependent release of endogenous APP intracellular domain (AICD) and inhibit neurogenesis in a concentration-dependent manner. ${ }^{20,21}$ These findings raise the possibility that CNTN2-APP interaction may play a similar role in GSCs, which could be a potential signaling pathway target for glioma therapy.

CNTN2 is a glycophosphatidylinositol (GPI)-linked neuronal membrane protein, a member of the immunoglobulin superfamily. CNTN2 as a cell adhesion molecule has been implicated in the axon growth and myelination. ${ }^{22-24}$ Its mutation is associated with cortical myoclonic tremor and epilepsy. Its association to epilepsy is attributed to the interaction of CNTN2 with contactin-associated protein-like 2 (CNTNAP2), which is necessary to maintain voltagegated potassium channels at the juxtaparanodal region. ${ }^{25}$ Aberrant expression of CNTN2 also correlates with glial tumorigenesis. ${ }^{26,27}$ Blocking CNTN2 expression can inhibit the migration of glioma cells. ${ }^{27} \mathrm{CNTN} 2$ may also promote the proliferation of granule cells in the cerebellum by regulating the sonic hedgehog (SHH) pathway. ${ }^{28}$ Since CNTN2 promotes proliferation in granule cells and inhibits neurogenesis in NSCs, we hypothesize that CNTN2 can stimulate proliferation of U87-GSCs, and CNTN2 RNA interference (RNAi) can inhibit the proliferation of U87-GSCs.

APP is an integral type I transmembrane protein widely distributed in the brain and involved in signal transduction, cell adhesion, and neuronal migration, differentiation, and regeneration. Its product $\beta$ amyloid is associated with Alzheimer's disease (AD). ${ }^{29}$ APP is cleaved by $\gamma$-secretase to produce AICD, which could regulate phosphoinositidemediated calcium signaling, ${ }^{29}$ inhibit neurogenesis, ${ }^{20,21}$ and inhibit Wnt signaling. ${ }^{30}$ APP expression is significantly increased in gliomas. ${ }^{31}$ Therefore, we hypothesize that the anti-proliferation function of CNTN2 RNAi involves the APP/AICD pathway in U87-GSCs.

Analogous to APP, Notch is another membrane receptor that is cleaved by $\gamma$-secretase to produce the Notch intracellular domain (NICD). ${ }^{32}$ The Notch/HES1 pro-proliferation pathway is activated in gliomas or GSCs, which may contribute to the radioresistance of GSCs. Inhibition of the Notch/HES1 pathway may induce cell autophagy. ${ }^{33-35}$ Cross talk between APP/AICD and Notch/NICD involves AICD overexpression, which activates Notch-targeting gene HES1. NICD overexpression activates APP-targeting gene KAI1. ${ }^{36}$ CD133+ GSCs have the characteristics of NSCs with neurospherelike growth and asymmetrical division, and they express all three lineages of neural markers: glial fibrillary acidic protein (GFAP), $\beta$-tubulin III, and O1. CD133+ GSCs comprise $1 \%-20 \%$ of glioblastomas. ${ }^{37}$ The CD133+ GSCs express more vascular endothelial growth factor (VEGF) than CD133- cells. ${ }^{38}$ Furthermore, the activation of EGFR/PI3K/ AKT pathway promotes the invasion of CD133+ GSCs $;{ }^{8}$ cross talk between APP/AICD and EGFR involves AICD binding to $E G F R$ promoter and regulates its expression. ${ }^{39}$ Based on the earlier observations, we hypothesize that the anti-proliferation function of CNTN2 RNAi may also affect the Notch/HES1 and EGFR/PI3K/AKT pathways in U87-GSCs by reducing the expression of HES1 and EGFR as downstream targets of APP/AICD and ultimately inhibiting GSC proliferation.

In this study, we first isolated and characterized U87GSCs and then showed the upregulation of CNTN2 and APP expressions in these cells. Next, we showed that CNTN2 RNAi could inhibit the proliferation of U87-GSCs and downregulate the AICD expression. Finally, we found that CNTN2 RNAi could also downregulate the expression of EGFR and HES1, which are downstream targets of AICD. This suggests that CNTN2 may stimulate the proliferation of U87-GSCs through activation of APP/AICD, Notch/HES1, and EGFR/ PI3K/AKT pathways; CNTN2 could be an ideal upstream target for the development of glioma therapy.

\section{Materials and methods GSC culture and characterization}

The human glioma cell line U87 (previously known as U87$\mathrm{MG})^{6}$ was purchased from the Cell Library of the Chinese Academy of Sciences (Shanghai, China). U87 cells were grown in Dulbecco's Modified Eagle's Medium (DMEM; HyClone, Utah, USA) supplemented with $10 \%$ fetal bovine serum in a humidified incubator with $5 \% \mathrm{CO}_{2}$ at $37^{\circ} \mathrm{C}$ and routinely passaged at 2- to 3 -day intervals. U87-GSCs 
were obtained through stemness-associated tumor sphere formation by culturing U87 cells in serum-free DMEM/F12 medium (HyClone) supplemented with 2\% B27 (Gibco, NY, USA), $20 \mathrm{ng} / \mathrm{mL}$ epidermal growth factor (EGF; PeproTech Inc., NJ, USA), and $20 \mathrm{ng} / \mathrm{mL}$ basic fibroblast growth factor (bFGF; PeproTech Inc.) with $\mathrm{pH}$ adjusted to 7.4. U87-GSCs were enriched by incubation in $1 \% \mathrm{O}_{2}$ tension, as described by Liu et al. ${ }^{40}$ The tumor spheres after the fifth passage (P5) were harvested for experiments.

Expression of GSC markers in tumor spheres was detected by immunocytochemistry and flow cytometry. Tumor spheres and differentiated cells were incubated in precoated 24-well culture plates. After washing with phosphate-buffered saline (PBS), the cells were fixed with 4\% paraformaldehyde and permeabilized with $0.1 \%$ Triton X-100 for $15 \mathrm{~min}$. After blocking for $1 \mathrm{~h}$ with $5 \%$ bovine serum albumin (BSA; Sigma-Aldrich, St. Louis, USA), the attached cells were incubated with primary antibodies at $4^{\circ} \mathrm{C}$ overnight. The primary antibodies included CD133 (1:200; Santa Cruz, LA, USA) and nestin (1:500; Millipore, Massachusetts, USA) for tumor spheres and $\beta$-tubulin III (1:1000; Millipore), GFAP (1:250; Millipore), and O1 (1:500; Millipore) for the differentiated cells. After the primary antibody incubation, the cells were washed with PBS three times and incubated with the corresponding secondary antibodies (horseradish peroxide [HRP]-anti-mouse 1:500 or HRP-anti-rabbit 1:500) for $30 \mathrm{~min}$ at room temperature. After development with diaminobenzidine, the nuclei were counterstained with Mayer's hematoxylin. Images were captured with a camera on the microscope (Olympus).

\section{RNAi, lentivirus construction, and infection}

To knockdown CNTN2 expression in U87-GSCs, we designed four RNAi target sequences for the human CNTN2 gene (NM_005076.3; Table 1). The annealed oligo sequences were cloned into the empty plasmid pcDNA ${ }^{\mathrm{TM}} 6.2-\mathrm{GW} /$ EmGFP-miR. Intact RNAi target sequences inserted into the expression vectors pcDNA 6.2-GW/EmGFP-miR-CNTN2RNAi-1/-2/-3/-4 were confirmed by sequencing. The interference plasmids and the packaging plasmids (Packaging Mix) were co-transfected into $293 \mathrm{~T}$ cells to make lentivirus carrying the CNTN2-RNAi or EGFP. The expression of EGFP was used to determine the titer and multiplicity of infection (MOI) to infect all cells, which was defined as an MOI of 100. An MOI of 100 was used in the next experiments.

U87-GSCs $\left(3 \times 10^{5}\right)$ were dissociated into single cells and transferred to T25 Corning cell culture flasks (Corning Incorporated, Corning, NY, USA) with the sphere-growing medium. After $24 \mathrm{~h}$, the cells were infected with lentivirus carrying RNAi or EGFP, and the medium was replaced $8 \mathrm{~h}$ after infection. After $48 \mathrm{~h}$, the cells were harvested for the next experiments. The effects of CNTN2-RNAi in U87-GSCs were tested by Western blot with antibody specific for CNTN2 (1:800; Santa Cruz). The infection efficiency of lentivirus for both control group (EGFP) and experimental group (CNTN2-RNAi) was the same $(100 \%)$, and the expression levels of EGFP normalized by glyceraldehyde-3-phosphate dehydrogenase (GAPDH) were the same for both groups (data not shown).

\section{Quantification of U87-GSC sphere diameters and numbers}

Ten days after the RNAi lentiviral infection, we counted the number and measured the diameters of the U87-GSC spheres in three randomly chosen fields containing spheres. Images were captured with a Leica DMIRE2 inverted phase-contrast microscope with $10 \times$ objective lens and analyzed by Image photo software.

\section{Cell counting kit 8 (CCK-8) assay for cell proliferation}

CCK-8 (Dojindo, Kumamoto, Japan) was used to detect cell proliferative activity in vitro. The U87-GSCs were dissociated into single cells, and they (200 cells/well) were plated into 96-well plates with $200 \mu \mathrm{L}$ of medium. After 48, 72, and $96 \mathrm{~h}$, CCK-8 assays were performed following the instructions of

Table I CNTN2 RNAi sequences

CNTN2-RNAi-IF

CNTN2-RNAi-IR

CNTN2-RNAi-2F

CNTN2-RNAi-2R

CNTN2-RNAi-3F

CNTN2-RNAi-3R

CNTN2-RNAi-4F

CNTN2-RNAi-4R
TGCTGTTCATCTTCCACCGATAGGTGGTTTTGGCCACTGACTGACCACCTATCTGGAAGATGAA CCTGTTCATCTTCCAGATAGGTGGTCAGTCAGTGGCCAAAACCACCTATCGGTGGAAGATGAAC TGCTGTCCACTTTGCGCCACTTGATCGTTTTGGCCACTGACTGACGATCAAGTCGCAAAGTGGA CCTGTCCACTTTGCGACTTGATCGTCAGTCAGTGGCCAAAACGATCAAGTGGCGCAAAGTGGAC TGCTGTTTGGTTGCATCTCGCACAGAGTTTTGGCCACTGACTGACTCTGTGCGATGCAACCAAA CCTGTTTGGTTGCATCGCACAGAGTCAGTCAGTGGCCAAAACTCTGTGCGAGATGCAACCAAAC TGCTGTCTTATAGCCGGTGACTGCAGGTTTTGGCCACTGACTGACCTGCAGTCCGGCTATAAGA CCTGTCTTATAGCCGGACTGCAGGTCAGTCAGTGGCCAAAACCTGCAGTCACCGGCTATAAGAC

Abbreviations: CNTN2, contactin 2; RNAi, RNA interference; F, forward; R, reverse. 
the manufacturer. The optical density (OD) was read by using a microplate reader (BIO-RAD 680) at $450 \mathrm{~nm}$.

\section{Real-time quantitative reverse transcription polymerase chain reaction (qRT-PCR)}

Total RNA was extracted using Trizol (Invitrogen). After purification, the total RNA $(1.0 \mu \mathrm{g})$ was reverse-transcribed into complementary DNA (cDNA) with a total volume of $20 \mu \mathrm{L}$ per well in a reverse transcriptase reaction system (Takara, USA). The cDNA was subjected to real-time PCR amplification by using SYBR Premix Ex Taq ${ }^{\mathrm{TM}}$ (Takara) and the indicated primer sequences (Table 2). The relative expression level of messenger RNA (mRNA) of the target genes (CT [threshold cycle]) was determined by normalizing against the expression level of GAPDH.

\section{Western blot}

Cells were lysed with a lysis buffer supplemented with protease inhibitor mixture (KEYGEN Biochemicals, Nanjin, China). Proteins in samples were measured, and equal amounts of proteins $(20 \mu \mathrm{g})$ were separated with sodium dodecyl sulfate polyacrylamide gel electrophoresis (SDS-PAGE) gels (10\%) and were transferred to polyvinylidene fluoride (PVDF) membrane. After blocking with 5\% BSA, Western blots were incubated with the primary antibodies: anti-CNTN2 antibody (1:800; Santa Cruz), anti-APP antibody (1:1000; Abcam, Cambridge, England), anti-EGFR antibody (1:200; Santa Cruz), and anti-HES1 antibody (1:200; Santa Cruz) at $4^{\circ} \mathrm{C}$ overnight. After rinsing, the blots were incubated with HRP-conjugated secondary antibodies (1:1000; Boster Biotechnology, Wuhan, China), and the enhanced chemiluminescence plus Western blotting detection system (Beyotime Biotechnology) was used to visualize the immune bands.

The detection of AICD by Western blot was as described by $\mathrm{Ma}$ et al. ${ }^{20}$ In brief, the proteins were extracted with $5 \times$ tricine sample buffer containing protease inhibitor mixture

Table 2 Primer sequences

\begin{tabular}{ll}
\hline APP-F & GTGGCTGAGGAGATTCAAG \\
APP-R & AAAGAAGGCATGAGAGCATC \\
CNTN2-F & AGTCACAGCCTGTCCTCTAG \\
CNTN2-R & ATCTGCCTATGCCTTGGTTG \\
EGFR-F & AACACAGTGGAGCGAATTCCTTT \\
EGFR-R & GGAAGTCCATCGACATGTTGCT \\
HESI-F & TTCTGGAAATGACAGTGAAGCA \\
HESI-R & CCTCGGTATTAACGCCCTC \\
GAPDH-F & TCATGGGTGTGAACCATGAGAA \\
GAPDH-R & GGCAGGACTGTGGTCATGAG
\end{tabular}

Abbreviations: APP, amyloid $\beta$ precursor protein; CNTN2, contactin 2; EGFR, epidermal growth factor receptor; $F$, forward; R, reverse.
(KEYGEN Biochemicals) and loaded on to $12 \%$ SDS-tricine polyacrylamide gel for electrophoresis after sonication and boiling. After transfer, the PVDF membranes were warmed by intermittent microwave irradiation $(5 \times 10 \mathrm{~s}$ irradiation at 5-min intervals) and processed for the subsequent procedures. The anti-APP C terminal antibody (1:2000, A8717; Sigma-Aldrich) was used as the primary antibody for AICD detection.

\section{Statistical analysis}

All experiments were repeated three times. The quantitative data were presented as mean \pm standard deviation (SD) for each group. Student's $t$-test was used to compare the mean in experiments with two groups. One-way analysis of variance (ANOVA) and Fisher's least significant difference (LSD) tests were used as post hoc for comparisons in experiments with more than two groups. If the homogeneity hypothesis failed, Dunnett's $\mathrm{t} 3$ test was used to analyze the significant differences between groups. Two-way ANOVA was used in the CCK-8 experiments. The significance level of all tests was set at $P \leq 0.05$. Statistical analysis was performed by using IBM SPSS Version 20 (SPSS Statistics V20; IBM Corporation, Somers, NY, USA).

\section{Results}

\section{Characterization of U87-GSCs}

Glioblastomas are composed of heterogenous population of cells, including GSCs and differentiated astrocytes. To target the GSCs in glioblastoma therapy, we first need to understand the molecular pathology of these cells; thus, a GSC model with a pure population of cells, true stemness, and easy enrichment is necessary. For this purpose, we harvested the U87-GSCs (suspending sphere culture of U87 cells) and increased the stemness by hypoxia conditioning. After 1 month and five passages of culture in serum-free medium and $1 \% \mathrm{O}_{2}$ tension, the U87-GSCs grew into suspending spheres with diameters $\sim 120 \mu \mathrm{m}$ (Figure 1A, phase contrast). These cells had healthy nuclei indicated by hematoxylin and eosin (HE) staining (Figure 1A, HE) and were mostly positive for CD133 and Nestin, the specific markers for GSCs or NSCs (Figure 1A, CD133, Nestin). The ratio of CD133+ and Nestin+ cells among total GSCs was $91.99 \% \pm 0.24 \%$ for U87-GSCs and $0.54 \% \pm 0.31 \%$ for U87 cells, as shown by the flow cytometry assay (Figure 1B). To identify the multilineage differentiation potential of the U87-GSCs, tumor spheres were cultured in serum-containing medium for 7 days and tested by immunocytochemistry with $\beta$-tubulin III, GFAP, and O1 antibodies, the markers for differentiated neurons, astrocytes, and oligodendrocytes, respectively. All three lineages of NSCs positively stained 

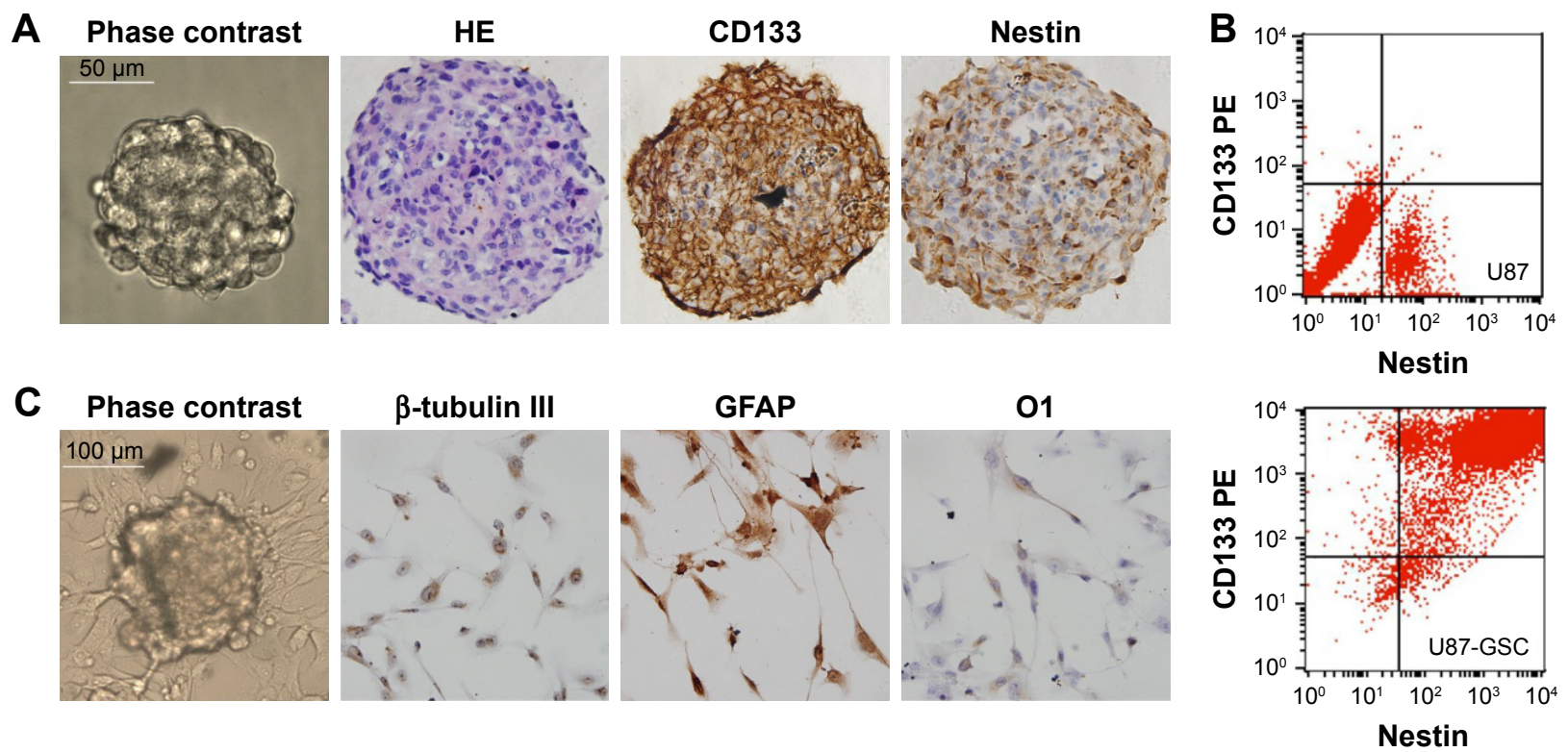

Figure I Characterization of U87-GSCs.

Notes: (A) U87-GSCs are cultured in serum-free medium for I month, and the P5 spheres are shown with phase-contrast, HE staining (violet), and immunostaining with GSC markers CDI 33 and Nestin (brown). (B) Flow cytometry showed that the percentage of CDI $33+/$ Nestin + cells were $0.540 \% \pm 0.308 \%$ and $91.993 \% \pm 0.238 \%$ in U87 cells (upper) and U87-GSCs (lower), respectively. (C) U87-GSCs are cultured in differentiation medium containing serum for 7 days, and the differentiated neurons, astrocytes, and oligodendrocytes are demonstrated by immunostaining with $\beta$-tubulin III, GFAP, and OI, respectively (brown). Scale bar $=50 \mu \mathrm{m}$ for (A) and $100 \mu \mathrm{m}$ for (C). Abbreviations: U87-GSCs, U87-derived glioma stem cells; HE, hematoxylin and eosin; GFAP, glial fibrillary acidic protein; PE, phycoerythrin.

cells in the differentiated U87-GSCs population (Figure 1C), suggesting the capability of U87-GSCs can differentiate into three neural cell lineages.

\section{Increased CNTN2 and APP expressions in GSCs-U87 cells}

As mentioned earlier, CNTN2 and APP expressions have been observed in primary glioma cells, established glioma lines, and NSCs; CNTN2-APP interaction regulates proliferation of NSCs. To investigate the possible involvement of CNTN2 and APP in the growth regulation of U87-GSCs, we first examined their expression in U87-GSCs cells by qRT-PCR and Western blot. CNTN2 and APP mRNA levels in U87-GSCs cells were significantly increased $(t=-3.581$, $P=0.023$, and $t=-4.913, P=0.039$, respectively) than those in U87 cells. Protein levels of CNTN2 and APP were also significantly increased $(t=-3.560, P=0.024$, and $t=-9.839$, $P=0.001$ ) (Figure 2). The change of CNTN2 ( 1-fold) was higher than that of APP ( 0.2-fold). These data showed that U87-GSCs supported higher CNTN2 and APP transcription and stable protein levels than U87 cells.

\section{CNTN2 RNAi inhibited AICD expression and proliferation in U87-GSCs}

Since CNTN2 binds APP, activates AICD, and inhibits neurogenesis in NSCs, the CNTN2-APP/AICD pathway may also exist in GSCs. To test the hypotheses that CNTN2 can stimulate proliferation of U87-GSCs and that CNTN2 RNAi can inhibit the proliferation of U87-GSCs through APP/AICD, we designed and engineered vectors that express one of four CNTN2 RNAi sequences. We tested their effects on selectively silencing CNTN2 expression in U87-GSCs. After infecting the U87-GSCs with lentivirus carrying one of four different CNTN2 RNAi sequences, we screened the most effective RNAi by Western blot. Compared to other CNTN2 RNAi vectors, CNTN2 RNAi-2-containing vector had the strongest silencing effect (Figure S1).

Thus, we used CNTN2 RNAi-2 to block the CNTN2 expression in U87-GSCs and observed its effects on AICD expression and sphere growth in these cells. As expected, the AICD expression in U87-GSCs was significantly downregulated by CNTN2 RNAi-2; however, the APP expression was not affected (Figure 3A). However, the growth of U87-GSC spheres was inhibited by CNTN2 RNAi-2, with reduced sphere number and size and a slower growth curve (Figure 3B). These results suggest that CNTN2 RNAi can inhibit the proliferation of GSCs and AICD expression, and CNTN2 may function through AICD to regulate proliferation in U87-GSCs.

\section{CNTN2 RNAi inhibited EGFR and HESI expressions in U87-GSCs}

To further investigate the mechanisms of CNTN2/APP/ AICD signaling in regulating the proliferation of U87-GSCs 
A
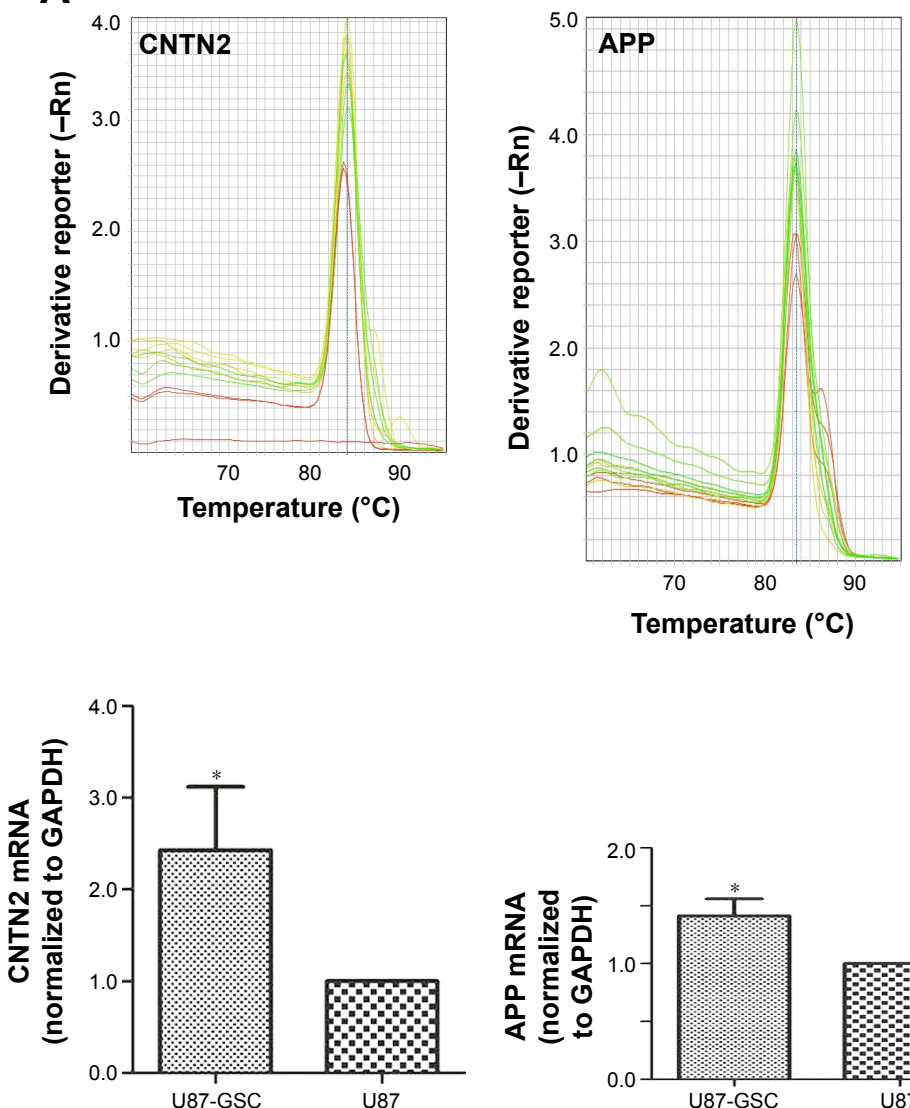

B
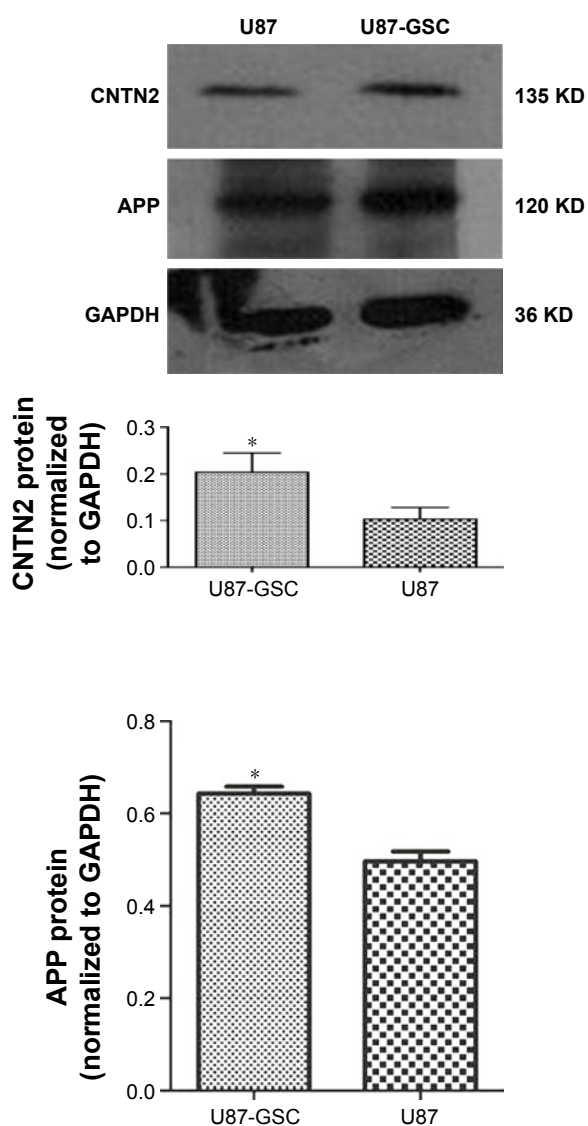

Figure 2 Expression of CNTN2 and APP in U87-GSCs and U87 cells.

Notes: (A) The melt curves of qPCR showed more CNTN2 (left) and APP (right) mRNA expression in U87-GSCs than those in U87 cells with the quantification graphs below. The different colored lines represent the different samples. (B) The Western blot demonstrated a higher CNTN2 and APP protein expression in U87-GSCs than those in $U 87$ cells with the quantification graphs below. $* P \leq 0.05$.

Abbreviations: CNTN2, contactin 2; APP, amyloid $\beta$ precursor protein; U87-GSCs, U87-derived glioma stem cells; GAPDH, glyceraldehyde 3-phosphate dehydrogenase.

and the potential downstream targets of AICD, we tested the hypothesis that CNTN2 RNAi can inhibit EGFR and HES1 expressions in U87-GSCs. Expression levels of EGFR and HES1 were both significantly downregulated as shown by qRT-PCR (Figure 4A) and Western blot (Figure 4B). These data suggest that CNTN2 may regulate proliferation of U87-GSCs through EGFR and HES1.

\section{Discussion}

CD133 is the most commonly used cell surface marker for GSCs. CD133+ cells from gliomas are capable of multilineage differentiation, have a high capacity for sphere formation, express significantly higher levels of NSC genes, and can form tumor after intracranial transplantation into mice. ${ }^{3}$ Increased expression of antiapoptotic genes in CD133+ GSCs may contribute to the resistance of GBM to chemotherapy, ${ }^{40}$ and Nestin+ GSCs are a subpopulation resistant to chemotherapy. ${ }^{16}$ In our model of U87-GSCs, the CD133+/Nestin+ cells comprised $\sim 90 \%$ of total cells; thus, it is a relatively purified population with characteristics of NSCs.
In this study, we used the standard sphere-forming method to grow U87-GSCs. The tumor spheres were enriched in serum-free culture supplemented with growth factors. There might be different populations of GSCs in the beginning of the culture, but after continuous passages, the faster subpopulation should overgrow the slower subpopulation in the microenvironment promoting growth. The $\mathrm{O}_{2}$ tensions in solid tumors can range from $0.1 \%$ to $5.3 \%$, with the lowest levels in the center region. ${ }^{41,42}$ Severe hypoxia can inhibit the proliferation, whereas mild hypoxia may promote the proliferation of GSCs. ${ }^{43}$ Mild hypoxia can increase VEGF and hypoxia-inducing factor (HIF) expression in GSCs, and it stimulates Notch signaling pathway: these three effects support the proliferation of GSCs. ${ }^{4}$ Our culture with $1 \%$ oxygen tension at mild range helped to promote the sphere growth of U87-GSCs, and we can enrich the spheres over five passages in 1 month. However, sphere culture is limited by increasing differentiation and cell death in the larger sizes of spheres. Laminin-coated culture could be an alternative choice. ${ }^{3}$ 
A
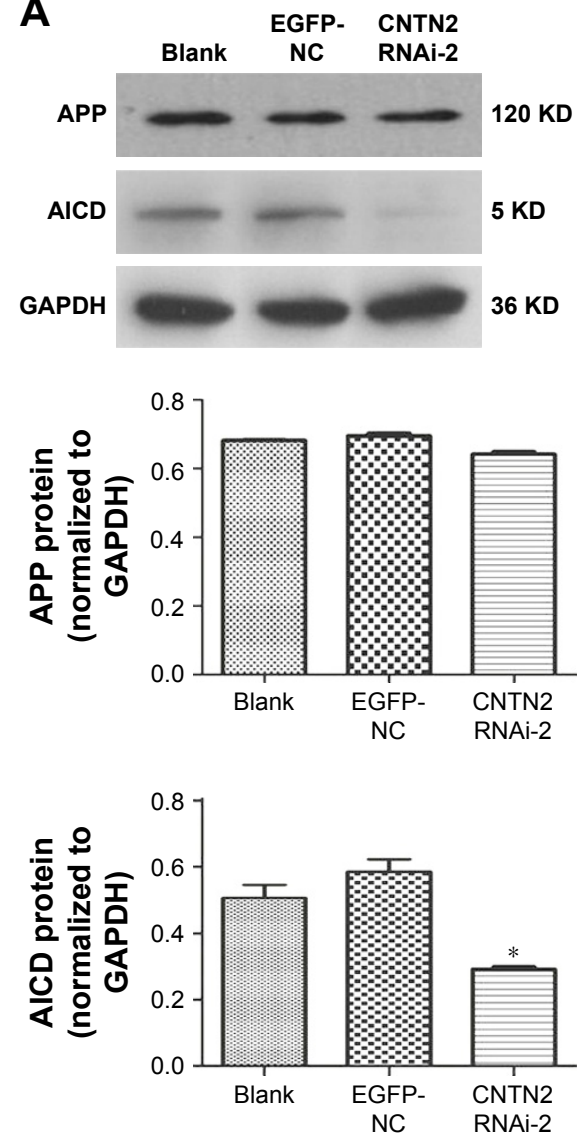

B
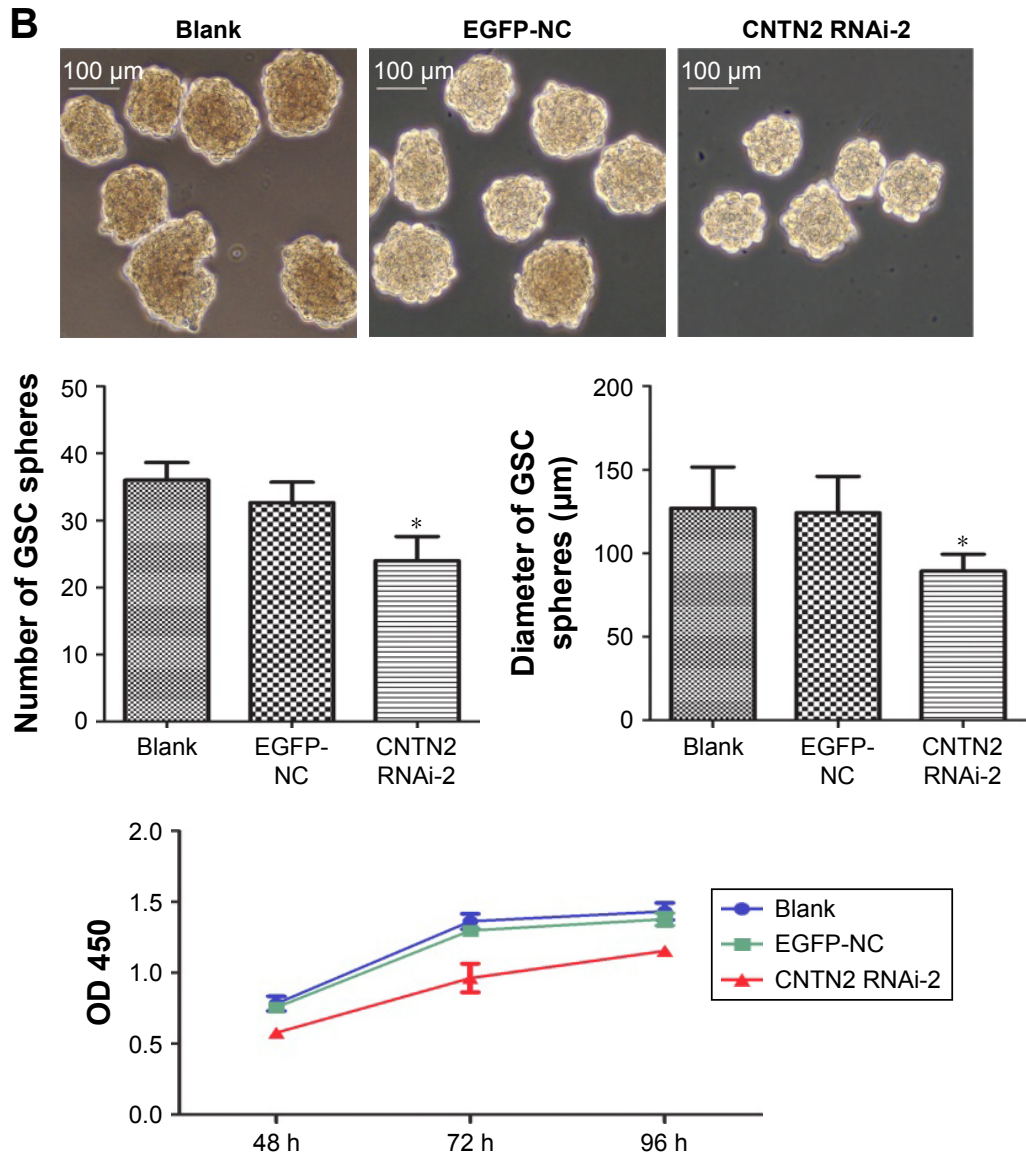

Figure 3 CNTN2 RNAi inhibited AICD expression and U87-GSCs proliferation.

Notes: (A) The Western blot demonstrates that lentiviral infection with CNTN2 RNAi-2 does not change the APP expression but significantly downregulates the AICD expression in U87-GSCs, with quantification graphs below. (B) The U87-GSCs were cultured for $72 \mathrm{~h}$ after the lentiviral infection with CNTN2 RNAi-2. The CNTN2 RNAi2-infected U87-GSCs showed lower proliferation by the phase-contrast micro-imaging (upper lane) with the quantifications of number and diameter of spheres (middle lane) and the time-dependent growth curves (lower lane). Scale bar $=100 \mu \mathrm{m}$ for (B). $* P \leq 0.05$.

Abbreviations: CNTN2, contactin 2; RNAi, RNA interference; AICD, APP intracellular domain; U87-GSCs, U87-derived glioma stem cells; APP, amyloid $\beta$ precursor protein; OD, optical density; GAPDH, glyceraldehyde 3-phosphate dehydrogenase.

In the GSCs model, GSCs rather than differentiated cells are the origins of tumorigenic cells. The proliferation and apoptosis of GSCs thus play an important role in the development of malignancy. Several signaling pathways involved in cell proliferation and apoptosis, such as Notch/ HES1 and EGFR/PI3K/AKT signaling pathways, have been proposed to be important in GSCs. ${ }^{8,33}$ Another closely related pathway, CNTN2/APP/AICD, has been found in NSCs, where CNTN2 as the functional ligand of APP can activate the release of downstream proteolytic signal AICD and inhibit neurogenesis. ${ }^{20,21}$ Therefore, we intended to examine these signaling pathways in our model of U87-GSCs.

Both CNTN2 and APP are expressed in gliomas. Rickman et $\mathrm{al}^{27}$ have reported that increased expression of CNTN2 was found both in middle- and high-grade glioma cells from clinical patients and in glioma cell lines. Culicchia et $\mathrm{al}^{31}$ demonstrated that APP expression levels were significantly higher in primary glioma cells and glioblastoma cell lines than in primary neuronal cells: the higher APP levels were considered to be related to abnormal proliferation and abnormal morphology of glioma cells. Consistent with these observations, we also found upregulation of CNTN2 and APP in U87-GSCs, with CNTN2 more significant. Xenaki et al ${ }^{28}$ found that CNTN2 promoted the proliferation and suppressed the differentiation of granule neuron progenitors through SHH signaling pathway. Consistent with this observation and that of Ma et al, ${ }^{20}$ we found that CNTN2 RNAi can inhibit the proliferation and growth of U87-GSCs. In contrast, overexpression of contactin did not affect the proliferation of glioblastoma cells. ${ }^{26}$ One possibility is that these glioblastoma cells are different compared to our U87-GSCs, which are stem cells. Alternatively, contactin has a distinct function compared to CNTN2: CNTN1 and CNTN2 may have opposite functions and play antagonistic roles on proliferation. ${ }^{28}$

AICD, as the downstream intracellular signaling CNTN2/ APP pathway, is considered to regulate the transcription 
A
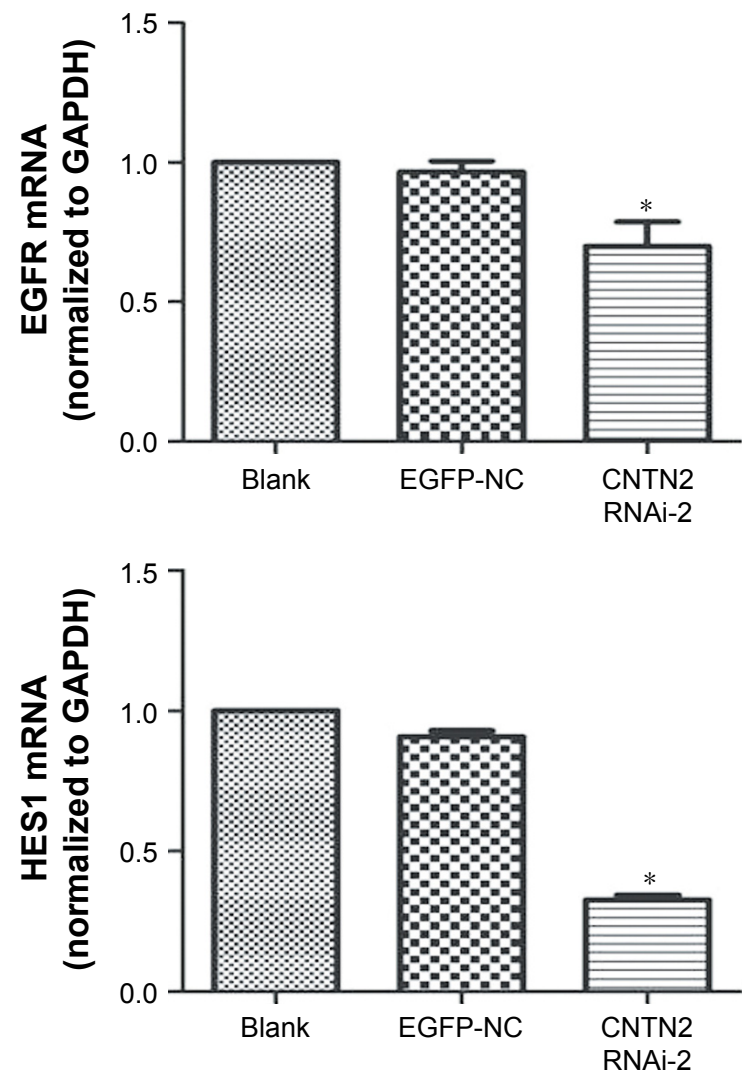

B

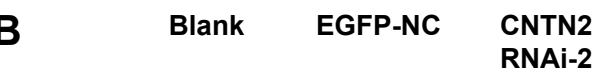

EGFR

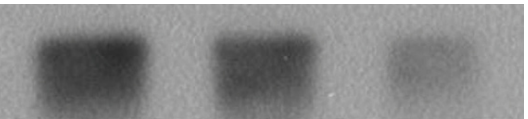

$170 \mathrm{kD}$

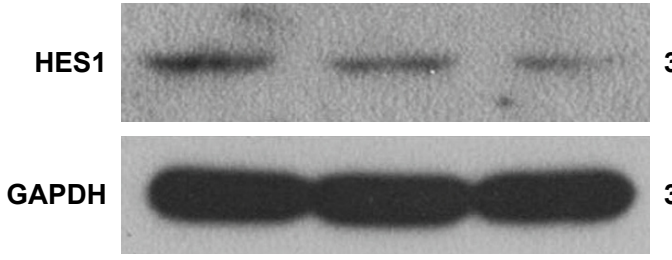

$35 \mathrm{kD}$
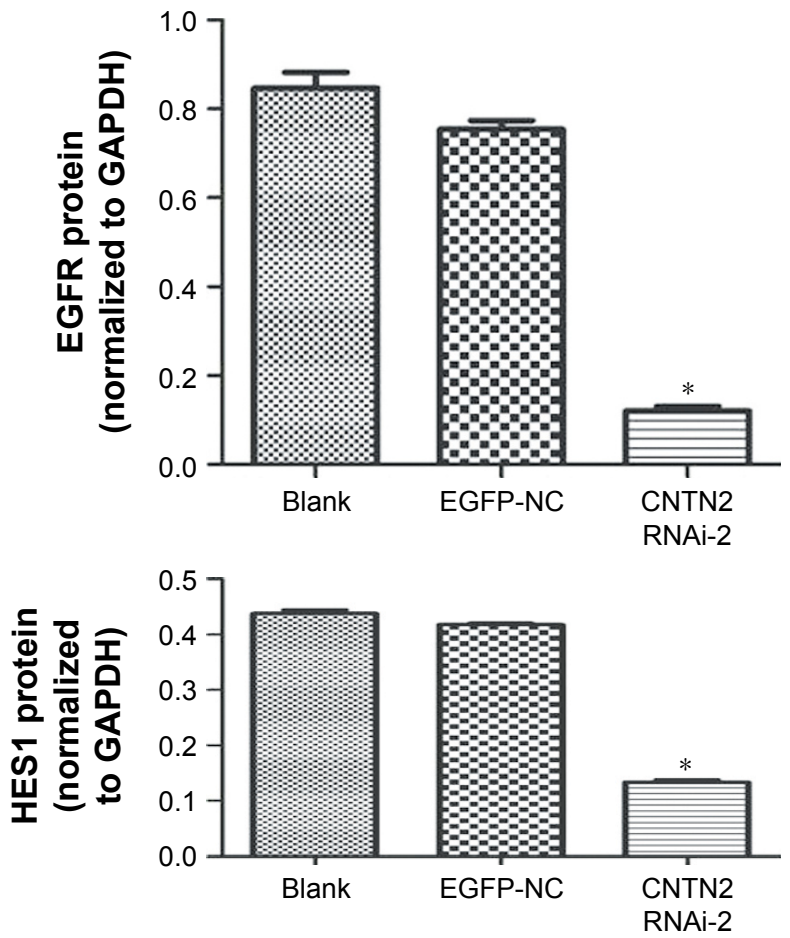

Figure 4 CNTN2 RNAi inhibited EGFR and HESI expressions in U87-GSCs.

Notes: (A) The quantification graphs of qPCR demonstrate that lentiviral infection with CNTN2 RNAi-2 significantly decreased EGFR and HESI mRNA levels in U87-GSCs. (B) The Western blot demonstrates that lentiviral infection with CNTN2 RNAi-2 significantly downregulated the EGFR and HESI protein expression in U87-GSCs, with quantification graphs below. $* P \leq 0.05$.

Abbreviations: CNTN2, contactin 2; RNAi, RNA interference; EGFR, epidermal growth factor receptor; U87-GSCs, U87-derived glioma stem cells; qPCR, quantitative polymerase chain reaction; mRNA, messenger RNA; GAPDH, glyceraldehyde 3-phosphate dehydrogenase.

of its target genes associated with cell proliferation and apoptosis within the nucleus. ${ }^{43}$ These nuclear targets may include EGFR promoter, or transcription factor HES1, as suggested by their involvement in Notch/HES1, and EGFR/ $\mathrm{PI} 3 \mathrm{~K} / \mathrm{AKT}$ signaling pathways in GSCs, ${ }^{8,33}$ and by HES1 expression induced by AICD overexpression. ${ }^{36}$ Our results expanded these earlier observations, where EGFR and HES1 were downregulated in U87-GSCs after CNTN2 RNAi treatment. A seemingly contradictory result is that CNTN2 RNAi decreased AICD but not APP expression, while APP was increased in U87-GSCs. CNTN2 RNAi decreased the CNTN2 protein, which is the ligand of APP. The APP level was not affected, only less ligand led to less activation of
APP and lower AICD production. These data indicate that CNTN2/APP/AICD/HES1 or CNTN2/APP/AICD/EGFR signaling pathway exists in U87-GSCs. However, the reduction of EGFR expression after CNTN2 RNAi treatment in U87-GSCs is not consistent with Zhang et al, ${ }^{39}$ where AICD overexpression decreased EGFR expression. ${ }^{39}$ Here are some possible explanations. 1) The study of Zhang et al investigated a mouse model that overexpressed AICD, and effects of AICD overexpression may be different in different cells. 2) EGFR signaling pathway, as an important signal of glioma genesis and proliferation, may be regulated by multiple signaling pathways, which may complicate the interpretation of observations in different models. For example, the cross 
talk between Notch/HES1 and APP/AICD pathways may also exist between Notch/HES1 and EGFR pathway. We need further experiments to confirm the effects of CNTN2 on EGFR signaling pathway.

Although our study on U87-GSC line elucidated that the CNTN2/APP/AICD/HES1 or CNTN2/APP/AICD/EGFR signaling pathway is involved in proliferation and can be inhibited by interfering RNA, a limitation of our study is that we investigated these pathways only in a single cell line. While we hypothesize that these pathways are activated in GSCs from other gliomas, these results may not be applicable to all GSCs from all gliomas. Our future studies will investigate the role of these and other pathways in additional cell lines.

\section{Conclusion}

Our results reveal for the first time that CNTN2/APP signaling can regulate the proliferation of U87-GSCs. CNTN2 RNAi inhibits the expression of AICD, HES1, and EGFR in U87-GSCs. At least partially, inhibition of CNTN2/APP signaling pathway may repress proliferation in U87-GSCs via downregulating the expression of HES1 and EGFR.

\section{Acknowledgment}

Yang Guo and Peidong Zhang are co-first authors.

\section{Disclosure}

The authors report no conflicts of interest in this work.

\section{References}

1. Ostrom QT, Gittleman H, Fulop J. CBTRUS statistical report: primary brain and central nervous system tumors diagnosed in the United States in 2008-2012. Neuro Oncol. 2015;17(suppl 4):iv1-iv62.

2. Galli R, Binda E, Orfanelli U, et al. Isolation and characterization of tumorigenic, stem-like neural precursors from human glioblastoma. Cancer Res. 2004;64(19):7011-7021.

3. Gilbert CA, Ross AH. Cancer stem cells: cell culture, markers, and targets for new therapies. J Cell Biochem. 2009;108(5):1031-1038.

4. Sundar SJ, Hsieh JK, Manjila S, Lathia JD, Sloan A. The role of cancer stem cells in glioblastoma. Neurosurg Focus. 2014;37(6):E6.

5. Cruz MH, Siden A, Calaf GM, Delwar ZM, Yakisich JS. The stemness phenotype model. ISRN Oncol. 2012;2012:392647.

6. Clark MJ, Homer N, O'Connor BD, et al. U87MG decoded: the genomic sequence of a cytogenetically aberrant human cancer cell line. PLoS Genet. 2010;6(1):e1000832.

7. Chen L, Chen XR, Chen FF, et al. MicroRNA-107 inhibits U87 glioma stem cells growth and invasion. Cell Mol Neurobiol. 2013;33(5): 651-657.

8. Chen X, Chen L, Chen J, et al. ADAM17 promotes U87 glioblastoma stem cell migration and invasion. Brain Res. 2013;1538:151-158.

9. Chen X, Chen L, Zhang R, et al. ADAM17 regulates self-renewal and differentiation of U87 glioblastoma stem cells. Neurosci Lett. 2013; $537: 44-49$

10. Kanno H, Sato H, Yokoyama TA, Yoshizumi T, Yamada S. The VHL tumor suppressor protein regulates tumorigenicity of U87-derived glioma stem-like cells by inhibiting the JAK/STAT signaling pathway. Int J Oncol. 2013;42(3):881-886.
11. Li P, Lu X, Wang Y, et al. MiR-181b suppresses proliferation of and reduces chemoresistance to temozolomide in U87 glioma stem cells. J Biomed Res. 2010;24(6):436-443.

12. Qiu B, Sun X, Zhang D, Wang Y, Tao J, Ou S. TRAIL and paclitaxel synergize to kill U87 cells and U87-derived stem-like cells in vitro. Int J Mol Sci. 2012;13(7):9142-9156.

13. Sassi Fde A, Caesar L, Jaeger M, et al. Inhibitory activities of trichostatin A in U87 glioblastoma cells and tumorsphere-derived cells. J Mol Neurosci. 2014;54(1):27-40.

14. Yu SC, Ping YF, Yi L, et al. Isolation and characterization of cancer stem cells from a human glioblastoma cell line U87. Cancer Lett. 2008;265(1):124-134.

15. Lamszus K, Gunther HS. Glioma stem cells as a target for treatment. Target Oncol. 2010;5(3):211-215.

16. Chen J, Li Y, Yu TS, et al. A restricted cell population propagates glioblastoma growth after chemotherapy. Nature. 2012;488(7412):522-526.

17. Wang J, Wakeman TP, Lathia JD, et al. Notch promotes radioresistance of glioma stem cells. Stem Cells. 2010;28(1):17-28.

18. Wei Y, Jiang Y, Zou F, et al. Activation of PI3K/Akt pathway by CD133-p85 interaction promotes tumorigenic capacity of glioma stem cells. Proc Natl Acad Sci U S A. 2013;110(17):6829-6834.

19. Zhou K, Song Y, Zhou W, et al. mGlu3 receptor blockade inhibits proliferation and promotes astrocytic phenotype in glioma stem cells. Cell Biol Int. 2014;38(4):426-434.

20. Ma QH, Bagnard D, Xiao ZC, Dawe GS. A TAG on to the neurogenic functions of APP. Cell Adh Migr. 2008;2(1):2-8.

21. Ma QH, Futagawa T, Yang WL, et al. A TAG1-APP signalling pathway through Fe65 negatively modulates neurogenesis. Nat Cell Biol. 2008;10(3):283-294.

22. Bizzoca A, Virgintino D, Lorusso L, et al. Transgenic mice expressing F3/contactin from the TAG-1 promoter exhibit developmentally regulated changes in the differentiation of cerebellar neurons. Development. 2003;130(1):29-43.

23. Falk J, Bonnon C, Girault JA, Faivre-Sarrailh C. F3/contactin, a neuronal cell adhesion molecule implicated in axogenesis and myelination. Biol Cell. 2002;94(6):327-334.

24. Fujita N, Saito R, Watanabe K, Nagata S. An essential role of the neuronal cell adhesion molecule contactin in development of the Xenopus primary sensory system. Dev Biol. 2000;221(2):308-320.

25. Stogmann E, Reinthaler E, Eltawil S, et al. Autosomal recessive cortical myoclonic tremor and epilepsy: association with a mutation in the potassium channel associated gene CNTN2. Brain. 2013;136(pt 4): $1155-1160$

26. Eckerich C, Zapf S, Ulbricht U, et al. Contactin is expressed in human astrocytic gliomas and mediates repulsive effects. Glia. 2006;53(1):1-12.

27. Rickman DS, Tyagi R, Zhu XX, et al. The gene for the axonal cell adhesion molecule TAX-1 is amplified and aberrantly expressed in malignant gliomas. Cancer Res. 2001;61(5):2162-2168.

28. Xenaki D, Martin IB, Yoshida L, et al. F3/contactin and TAG1 play antagonistic roles in the regulation of sonic hedgehog-induced cerebellar granule neuron progenitor proliferation. Development. 2011;138(3): 519-529.

29. Leissring MA, Murphy MP, Mead TR, et al. A physiologic signaling role for the gamma-secretase-derived intracellular fragment of APP Proc Natl Acad Sci U S A. 2002;99(7):4697-4702.

30. Zhou F, Gong K, Song B, et al. The APP intracellular domain (AICD) inhibits Wnt signalling and promotes neurite outgrowth. Biochim Biophys Acta. 2012;1823(8):1233-1241.

31. Culicchia F, Cui JG, Li YY, Lukiw WJ. Upregulation of beta-amyloid precursor protein expression in glioblastoma multiforme. Neuroreport. 2008;19(9):981-985.

32. Shimojo H, Ohtsuka T, Kageyama R. Dynamic expression of notch signaling genes in neural stem/progenitor cells. Front Neurosci. 2011;5:78.

33. Kanamori M, Kawaguchi T, Nigro JM, et al. Contribution of Notch signaling activation to human glioblastoma multiforme. J Neurosurg. 2007;106(3):417-427. 
34. Shen Y, Chen H, Zhang J, et al. Increased Notch signaling enhances radioresistance of malignant stromal cells induced by glioma stem/ progenitor cells. PLoS One. 2015;10(11):e0142594.

35. Yao J, Zheng K, Li C, Liu H, Shan X. Interference of Notch1 inhibits the growth of glioma cancer cells by inducing cell autophagy and down-regulation of Notch1-Hes-1 signaling pathway. Med Oncol. 2015; 32(6):610.

36. Fischer DF, van Dijk R, Sluijs JA, et al. Activation of the Notch pathway in Down syndrome: cross-talk of Notch and APP. FASEB J. 2005;19(11): 1451-1458.

37. Kahlert UD, Bender NO, Maciaczyk D, et al. CD133/CD15 defines distinct cell subpopulations with differential in vitro clonogenic activity and stem cell-related gene expression profile in in vitro propagated glioblastoma multiforme-derived cell line with a PNET-like component. Folia Neuropathol. 2012;50(4):357-368.

38. Bao S, Wu Q, Sathornsumetee S, et al. Stem cell-like glioma cells promote tumor angiogenesis through vascular endothelial growth factor. Cancer Res. 2006;66(16):7843-7848.
39. Zhang YW, Wang R, Liu Q, Zhang H, Liao FF, Xu H. Presenilin/gammasecretase-dependent processing of beta-amyloid precursor protein regulates EGF receptor expression. Proc Natl Acad Sci U S A. 2007; 104(25):10613-10618.

40. Liu G, Yuan X, Zeng Z, et al. Analysis of gene expression and chemoresistance of CD133+ cancer stem cells in glioblastoma. Mol Cancer. 2006;5:67.

41. Ljungkvist AS, Bussink J, Kaanders JH, van der Kogel AJ. Dynamics of tumor hypoxia measured with bioreductive hypoxic cell markers. Radiat Res. 2007;167(2):127-145.

42. Persano L, Rampazzo E, Della Puppa A, Pistollato F, Basso G. The three-layer concentric model of glioblastoma: cancer stem cells, microenvironmental regulation, and therapeutic implications. Scientific World Journal. 2011;11:1829-1841.

43. Konietzko U. AICD nuclear signaling and its possible contribution to Alzheimer's disease. Curr Alzheimer Res. 2012;9(2):200-216. 


\section{Supplementary material}
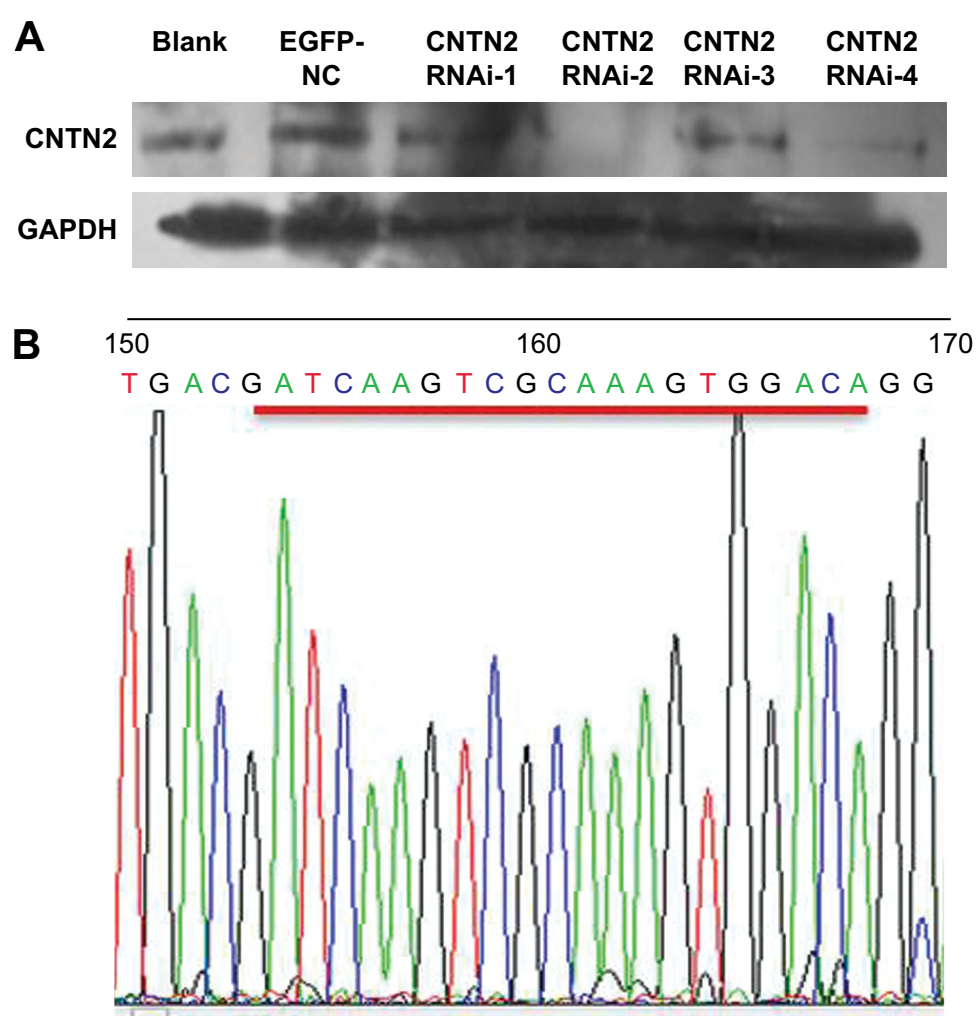

Figure SI CNTN2 RNAi selection.

Notes: (A) The Western blot shows that CNTN2 RNAi-2 was the most effective among the four RNAi vectors to inhibit CNTN2 expression in U87-GSCs. (B) Gene sequencing results of CNTN2 RNAi-2 after lentiviral infection of U87-GSCs.

Abbreviations: CNTN2, contactin 2; RNAi, RNA interference; U87-GSCs, U87-derived glioma stem cells; GAPDH, glyceraldehyde 3-phosphate dehydrogenase.

\section{Publish your work in this journal}

OncoTargets and Therapy is an international, peer-reviewed, open access journal focusing on the pathological basis of all cancers, potential targets for therapy and treatment protocols employed to improve the management of cancer patients. The journal also focuses on the impact of management programs and new therapeutic agents and protocols on patient perspectives such as quality of life, adherence and satisfaction. The manuscript management system is completely online and includes a very quick and fair peer-review system, which is all easy to use. Visit http://www.dovepress.com/testimonials.php to read real quotes from published authors. 